\title{
http:// bjas.bu.edu.eg \\ Fractional CO2 laser Assisted Delivery of Topical Tranexamic Acid in Treatment of Melasma
}

\author{
H.H.Sabry, R.M.Salemand A.M.Elkholy \\ Dermatology and Andrology Dept, Faculty of Medicine, Benha Univ, Benha, Egypt \\ E-Mail: derma.drabeer@gmail.com
}

\begin{abstract}
Increased pigmentation is the main characteristic of melasma. Melasma lesions have greater vascularization , solar elastosis and an increased number of fibroblasts and mast cells compared with perilesional normal skin, suggesting that altered dermal structures play an important role in the development of melasma. The aim of the present study was to compare the efficacy and safety of topically applied tranexamic acid in combination with Fractional Co2 laser verus Fractional Co2 only in the treatment of melasma. This study was conducted on 20 patients suffering from melasma. Patients were recruited from Dermatology outpatient clinic in Benha University. Written informed consents were obtained from all participants. The patients were divided into two groups.Group A in whom the whole face was treated with fractional CO2 laser followed by topical application of tanexamic acid solution, while group B were treated with fractional $\mathrm{CO} 2$ laser only. 50\% of the cases were in group A and 50\% were in group B. There is no significant difference between the studied groups as regard baseline data. Regarding the treatment efficacy, the results in both treatment groups were comparable. This table shows that there is no significant difference between the two studied groups as regard patient satisfaction. All patients [100\%] reported very mild burning pain after the sessions that lasted for few hours. Mild erythema was experienced by patients for 3-5 days following treatment sessions. Addition of topical tranexamic acid following fractional CO2 laser did not add to its efficacy.
\end{abstract}

Keywords: Melasma, TXA Fractional Co2 Laser.

\section{Introduction}

Melasma is a hyperpigmented lesion occurring alongside sun-exposed skin. increased pigmentation is the primary mark of melasma [1]. It additionally demonstrated that melasma lesions have more vascularization, solar elastosis also a expanded number for fibroblasts and mastcells compared with normal perilesional skin, suggesting that modified dermal structures assume the most important part in prognosis of melasma [2].

Tranexamic acid [TXA, trans-4-aminomethyl cyclohexane carboxylic acid], An plasmin inhibitor and antifibrinolytic has shown very good results in treatment of melasma. An oral, topical and microinjection forms o TXA have been used to enhance melasma treatment[3].

In spite of the fact that the mechanism about action of TXA stays unclear, it is suggested that it inhibits melanin synthesis. Furthermore It might have been Additionally proposed that TXA might reverse the abnormal dermal structures occured in melasma [4]. TXA has hypopigmentory impact as it inhibits plasmin and decrease the levels of prostanoid Furthermore alpha melanocyte-stimulating hormone [5].

Fractional resurfacing $[\mathrm{FR}]$ is a tool of skin rejuvenation that that produces a unique thermal damage pattern. FR produces multiple columns of thermal damage, referred to as microthermal treatment zones (MTZ), and spares the tissue surrounding each column. The elimination of the damaged epidermis containing the pigment in the basal cell layer facilitated by the movement of the rapidly migrating viable keratinocytes present at the wood margins. This process might explain why FR might provide a very good tool in the treatment of melasma. The histology of a microthermal treatment zone (MTZ) shows homogenization of dermal matrix and the formation of microscopic epidermal necrotic debris
(MEND). MENDs represent the elimination of the damaged epidermis containing the pigment in the basal cell layer facilitated by the movement of the rapidly migrating viable keratinocytes present at the wound margins. This process might explain why FR might provide a promising modality in the treatment of melasma[6].

\section{Patients and Methods}

The study was conducted on 20 patients suffering from melasma. Patients were recruited from Dermatology outpatient clinic of Benha University in the period of [Jan 2018-June 2019]. The studied population were divided into two groups .Group $\mathrm{A}$ in whom the whole face was treated with fractional $\mathrm{CO} 2$ laser followed by topical application of tanexamic acid solution, while group B were treated with fractional $\mathrm{CO} 2$ laser only.

\subsection{The treatment plan}

- The face was cleansed with alcohol.

- One hour before the procedure a local anesthetic, $30 \%$ topical lidocaine ointment was applied.

- Full face was treated with fractional $\mathrm{CO} 2$ laser for group $\mathrm{A}$ and $\mathrm{B}$.

- In patients of group A topical TXA solution $[100 \mathrm{mg} / \mathrm{ml}]$ was applied on the full face

- Session were repeated every month for 6 months.

\subsection{Inclusion criteria}

All patients enrolled in the study had:

- Clinically typical melasma.

- Different degrees of severity of melasma.

\subsection{Exclusion criteria}


- Pregnant, nursing women and women on contraceptive pills at the time of the study.

- Patients taking any photosensitizing drugs at the time of the study.

- Having any bleeding disorders or taking any kind of anticoagulants.

- Using any therapy for melasma during the study or 1 month before.

\subsection{Each patient was subjected to the following}

Full history taking: Including personal and melasma history as well as other skin diseases or drug intake.

\subsection{Clinical examination}

$>$ Complete cutaneous examination: Clinical assessment of melasma lesions were done to determine the distribution, clinical variants and the extent of melasma.

$>$ Digital photographs were taken before the treatment beginning and before each session.

\section{Results \\ 3.1Distribution of cases on treatment groups}

$50 \%$ of the cases were in group A and $50 \%$ were in group B. There is no significant difference between the studied groups as regard baseline data Table (1).

Regarding the treatment efficacy, the results in both treatment groups were comparable Table [2]. This table shows that there is no significant difference between the two studied groups as regard patient satisfaction.

\subsection{Assessment of Complications among the studied groups}

All patients [100\%] reported very mild burning pain after the sessions that lasted for few hours. Mild erythema was experienced by patients for 3-5 days following treatment sessions.

Table (1) Baseline data of the studied groups

\begin{tabular}{lccccccc}
\hline & & \multicolumn{3}{c}{ Treatment group } & \multicolumn{2}{c}{ T-Test or Chi-Square } \\
\cline { 3 - 7 } & & Group A & Group B & T or X & P-value \\
\hline \multirow{2}{*}{ Family history } & No & 10 & 50.00 & 7 & 70.00 & \multirow{2}{*}{1.086} & 0.297 \\
& Yes & 10 & 50.00 & 3 & 30.00 & & \\
\multirow{2}{*}{ Past Cosmetic } & Whiting cream & 16 & 80.00 & 5 & 50.00 & & \multirow{2}{*}{0.228} \\
& Chemical peeling & 2 & 10.00 & 3 & 30.00 & 2.957 & \\
& Laser resurfacing & 2 & 10.00 & 2 & 20.00 & & \\
\hline
\end{tabular}

Table (2) Improvement evaluation of patients

\begin{tabular}{|c|c|c|c|c|c|c|}
\hline \multirow{2}{*}{$\begin{array}{l}\text { Improvement } \\
\text { evaluation }\end{array}$} & \multicolumn{4}{|c|}{ Treatment group } & \multicolumn{2}{|c|}{ Chi-Square } \\
\hline & \multicolumn{2}{|c|}{ Group A } & \multicolumn{2}{|c|}{ Group B } & $\mathbf{X}^{2}$ & P-value \\
\hline Fair & 2 & 20.00 & 3 & 30.00 & & \\
\hline Poor & 2 & 20.00 & 3 & 30.00 & & \\
\hline Good & 4 & 40.00 & 3 & 30.00 & 2.518 & 0.472 \\
\hline Excellent & 2 & 20.00 & 1 & 10.00 & & \\
\hline Total & 10 & 100.00 & 10 & 100.00 & & \\
\hline
\end{tabular}

\section{Discussion}

Melasma, also referred to as chloasma, is a common acquired condition of hyperpigmentation marked by irregular hyperpigmented macules or patches and most commonly occurs in females and few number of males with dark skin types living in areas of intense ultraviolet (UV) light exposure. The prevalence of melasma ranges from $8.8 \%$ to $40 \%$ [7].

Numerous medications have been suggested to treat melasma including topical hydroquinone preparations, chemical peels and physical medication. Although some of these therapeutic medication achieved great effect in managing this challenge, their use might be limited by their side effects which include irritant dermatitis, allergic contact dermatitis, post-inflammatory hyperpigmentation, and exogenous ochronosis [8], and also the high costs of these treatments[9].
The aim of the present study was to compare the efficacy and safety of topically applied TXA in combination with fractional $\mathrm{CO} 2$ laser versus fractional $\mathrm{CO} 2$ laser in the treatment of melasma.

The study was conducted on 20 patients suffering from melasma. Patients were recruited from Dermatology outpatient clinic of Benha University in the period of (December 2018-June 2019). The studied populations were divided into two groups. Group A in whom the face was treated with fractional $\mathrm{CO} 2$ laser then topical tranexamic acid application, while group B was treated with fractional $\mathrm{CO} 2$ laser only. The present study showed that fractional $\mathrm{CO} 2$ laser

provided measurable improvement in the clinical appearance of melasma.

Previously, a prospective investigator-blinded consider by [10] in which 20 brazilian patients accepted 
$10 \mathrm{CO} 2$ laser showed diminished mMASI scores significally following the last session. C.Y.Hsiao [11] conducted a study to compare the skin histology and permeation of tranexamic acid after fractional and conventional $\mathrm{CO} 2$ laser treatment, found that Fractional laser treatment is as effective as conventional laser treatment in enhancing tranexamic acid delivery and causes less skin damage. E.Del Rosario [12] led a randomized, placebo-controlled, double blinded study to evaluate the efficacy of oral TXA in patients with moderate to severe melasma and noticed that at 3 months, there was a $49 \%$ reduction in mMASI score in the TXA group vs. $18 \%$ in the control group.

M.Banihashemi [13] who directed a split face trial on liposomal tranexamic acid and compared it to hydroquinone in melasma, discovered that the mean MASI scores significantly reduced in both treated sides after 12 week. A greater decrease was observed with 5\% liposomal TXA, although this difference was not statistically significant.

L.Budamakuntla [14] in prospective randomized open label study, compared the effect of TXA microinjections using a syringe and TXA micro-needling using microneedles in patients with melasma. They reported better improvement in patients treated with micro-needing than microinjections but this difference was not statistically significant. They concluded that TXA intra-lesional microinjection and micro-needling are an office- based procedures with relatively quick results, no significant side effects, and almost no downtime.

In a study by [15] who conducted a double-blind splitface trial to evaluate the efficacy and safety of topical solution of TXA and compare it with combined solution of hydroquinone and dexamethasone for melasma in Iranian women found decreasing in MASI score of both groups with no significant difference between them at the end of the study.

L.R.Sklar [16] studied experimentally the usefulness of laser in achieving transdermal systemic delivery of drugs with low oral bioavailability, transdermal delivery of vaccine and transdermal delivery of mesenchymal stem cells to the bone marrow.

$\mathrm{Na}$ Kanechorn [17] reported the patient satisfaction in their study of topical TXA solution for melasma treatment as excellent in one patient, moderate in 8 patients ,marginal in 5 patients, while 2 achieved no improvement at all.

S.Wu [18] demonstrated that when tranexamic acid was applied topically to the entire facial area, it lightened areas of melasma, but not other pigmented areas such as senile lentigo or freckles.

S.N.Abadchi [19] compared the efficacy of fractional $\mathrm{CO} 2$ laser in combination with topical therapy (hydroquinone) to topical therapy alone, concluded that considering the short-term outcome of laser and hydroquinone therapy, they could apply it to obtain earlier positive results. However, because of the lack of significant difference between the two methods and also the high cost of laser therapy, it seems better not to recommend fractional $\mathrm{CO} 2$ laser to patients as adjunctive therapy for long-term treatment of melasma.

M. A.Trelles, [20] assigned 30 female patients with melasma to one of three treatment groups: topical melasma cream (Kligman's formula), $\mathrm{CO} 2$ ablative fractional resurfacing, and a combination (laser and daily topical melasma cream). The results of the patients who underwent combined treatment withCO2 laser and longterm topical lightening cream showed the greatest improvement and were able to maintain the treatment benefits up to the 12-month post-treatment. Patients in the other groups were unable to sustain their initial improvement.

In the present study there was no significant difference between the two studied groups as regard treatment efficacy. Also we noticed that there was no significant difference between the studied groups as regard complications.

On the contradict, the largest retrospective study of TXA treatment was conducted in Singapore [21]. The authors reviewed data from 561 patients with melasma who were treated with TXA, and improvement was noted in $90 \%$ of patients. Adverse events were reported in 40 patients (7.1\%), and most side effects were mild. However, one patient developed deep vein thrombosis and was later discovered to have familial protein $S$ deficiency. There was a personal history of spontaneous miscarriage and a family history of thromboembolic issues in two siblings.

Angsuwarangsee and Polnikorn. [22] performed a split-face trial to study the efficacy of a Q-switched alexandrite 755-nm laser with or without one pass of an ultra-pulsed $\mathrm{CO} 2$ laser in six female patients with refractory melasma. Three patients developed $\mathrm{PIH}$ on both sides of the face 2 to 4 weeks post procedure and one patient developed hypopigmentation. Given the risk of postoperative dyspigmentation, the authors concluded that neither modality was safe enough to recommend for routine use to treat patients with melasma.

The current study showed that $100 \%$ of both groups both sides showed improvement with no significant difference between the studied groups.

A.Tourlaki [23] also verified that although they observed remarkable improvement in darkness during the first 3 weeks after combination therapy TCC (hydroquinone $4 \%$,retinoic acid $0.03 \%$, hydrocortisone $0.1 \%$ ) applied daily for 10 days followed by 4 fractional co2 laser session which performed in 3weeks interval during this interval and 3 months after last session ,TCC was applied. its long-term improvement was limited. At the end of the treatment, they observed $>75 \%$ improvement in only $5.4 \%$ of the patients and $75.67 \%$ of the patients had $<50 \%$ improvement with combination therapy.

In the current study, patients were satisfied by the results with no statistically difference between two groups denoting that fractional co2 laser with or without TXA is an effective lines of treatment of melasma. 


\section{Conclusion}

Fractional co2 laser is effective, safe treatment for melasma . topical tranexamic acid didn't add to the efficacy.

\section{References}

[1] W.H.Kang, K.H.Yoon, E.S.Lee, Melasma:histopathologicalcharacteristics in 56 Korean patients,Br J Dermatol, Vol. 146,PP.228$37,2002$.

[2] H.Y.Kang, J.P.Ortonne, What should be considered intreatment of melasma, Ann Dermatol, Vol.22,PP. $373-8,2010$.

[3] J.H.Lee, J.G.Park, S.H.Lim, Localized intradermal microinjection of tranexamic acid for treatment of melasma in Asian patients: a preliminary clinical trial, Dermatol Surg, Vol. 32,PP.626-31,2006.

[4] J.I. Na, S.Y.Choi, Effect of tranexamic acid on melasma: a clinical trial with histological evaluatio, J.,the European Academy of Dermatology and Venereology, Vol.27[8],PP.1035-1039,2013.

[5] H.Ando, M.S.Matsui, M.Ichihashi, Quasi-drugs developed in Japan for the prevention or treatment of hyperpigmentary disorders, Int J Mol Sci,Vol.11PP.2699-700,2014.

[6] D.Manstein, G.S.Herron, R.K.Sink, H.Tanner, R.R.Anderson,Fractional photothermolysis: a new concept for cutaneous remodeling using microscopic patterns of thermal injury, Lasers Surg Med ,Vol.34[5],PP.426-38,2004.

[7] A.G.Pandya, L.S.Hynan, R.Bhore, Reliability assessment and validation of the Melasma Area and Severity Index [MASI] and a new modified MASI scoring method,J Am Acad Dermatol,Vol. 64,PP. 78-83,2011.

[8] M.Perper, A.E.Eber, R.Fayne, S.H,Tranexamic acid in the treatment of melasma: a review of the literature, American J.,clinical dermatology, Vol.18[3],PP.373-381,2017.

[9] B.Desmedt, P.Courselle, J.O.De Beer, V.Rogiers , Overview of skin whitening agents with an insight into the illegal cosmetic market in Europe, J.,the European Academy of Dermatology and Venereology,Vol.30[6], PP. 943-950,2016.

[10] C.P.Hofbauer, M.F.Careta, N.Y.Valente, L.A. Torezan, Clinical and Histopathologic Assessment of Facial Melasma After Low-Fluence Q-Switched Neodymium-Doped Yttrium Aluminium Garnet Laser, Dermatologic surgery, official publication for American Society for Dermatologic Surgery, Vol. 42[4],PP.507-512,2016.

[11] C.Y.Hsiao, H.C.Sung, Y.L.Huang, Fractional CO2 laser pretreatment facilitates transdermal delivery of two

vitaminCderivatives Molecules, Vol. 21[1],PP.1547,2 016.

[12] E.Del Rosario, S.Florez-Pollack, J.r.L. Zapata, K.Hernandez , Randomized, placebo-controlled, double-blind study of oral tranexamic acid in the treatment of moderate-to-severe melasma. J.,the American Academy of Dermatology, Vol.78[2],PP. 363-369,2018

[13] M.Banihashemi, N.Zabolinejad, M.R.Jaafari, M.Salehi, Comparison of therapeutic effects of liposomal tranexamic acid and conventional hydroquinone on melasma, J.,cosmetic dermatology,Vol.14[3],PP.174-177,2015.

[14] L.Budamakuntla, E.Loganathan, D.H.Suresh, Q.Zhao, A Randomised open labelled study of Tranexamic Acid with microneedling in patient with melasma,J Cutan Aesthet Surg, Vol.6[3], PP.139143, 2013.

[15] B.Ebrahimi, F.F.Naeini, Topical tranexamic acid as a promising treatment for melasma, J.,research in medical science, the official J.,Isfahan University of Medical Sciences,Vol. 19[8],PP.753-68,2014.

[16] L.R.Sklar, C.T.Burnett, J.S.Waibel, R.L.Moy , Laser assisted drug delivery: a review of an evolving technology, Lasers in surgery and medicine,Vol.46[4], PP. 249-262,2014.

[17] Na.Kanechorn Ayuthaya, N.Niumphradit, A.Manosroi, A.Nakakes , Topical 5\% tranexamic acid for the treatment of melasma in Asians: a double-blind randomized controlled clinical trial, J.,Cosmetic and Laser Therapy, vol .14[3], PP.150-154,2012.

[18] S.Wu , H.Shi , L.Pan, Treatment of melasma with oral administration of tranexamic acid. Aesthetic plastic surgery, Vol. 36[4], PP.964-970,2012.

[19] S.N.Abadchi,F.F.Naeini,E.Beheshtian,Combination of hydroquinone and fractional $\mathrm{CO} 2$ laser versus hydroquinone monotherapy in melasma treatment, A randomized, single-blinded, split-face clinical trial, Indian J.,dermatology,Vol.64[2], PP. 129,2019.

[20] M. A.Trelles, M.Velez, , M.H.Gold,The treatment of melasma with topical creams alone, $\mathrm{CO} 2$ fractional ablative resurfacing alone, or a combination of the two: a comparative study, J.,drugs in dermatology,Vol. 9[4],PP.315-322,2014.

[21]H.C.Lee, T.Thng, C.L. Goh : Oral tranexamic acid [TXA] in the treatment of melasma: a retrospective analysis, J.,the American Academy of Dermatology,Vol. 75[2],PP. 385-392.2016.

[22] S.Angsuwarangsee, N.Polnikorn, Combined ultrapulse CO2 laser and Q-switched alexandrite laser compared with Q-switched alexandrite laser alone for refractory melasma: split-face design, Dermatologic surgery, Vol. 29[1],PP. 59$64,2003$.

[23] A.Tourlaki, M.G.Galimberti, G.Pellacani, P.L.Bencini, Combination of fractional erbium-glass laser and topical therapy in melasma resistant to triple-combination cream, J.,Dermatological Treatment,vol . 25[3],PP. 218-222,2014. 